\title{
TWO DIMENSIONAL EINSTEIN-WEYL STRUCTURES
}

\author{
DAVID M. J. CALDERBANK \\ Department of Mathematics and Statistics, University of Edinburgh, King's Buildings, Mayfield Road, \\ Edinburgh EH9 3JZ, Scotland \\ e-mail:davidmjc@maths.ed.ac.uk
}

\begin{abstract}
All local solutions of the two dimensional Einstein-Weyl equations are found, and related to the compact examples obtained in [1].
\end{abstract}

1991 Mathematics Subject Classification. 53A30, 53C25, 30F45.

Introduction. Einstein-Weyl geometry has received much attention in recent years $[\mathbf{2 , 4}]$, particularly in three dimensions [5,7], where Einstein-Weyl structures arise as symmetry reductions of the self-duality equations for four dimensional conformal structures [6]. An Einstein-Weyl structure on an $n$-manifold $M$, with $n \geq 3$, consists of a conformal structure together with a compatible (i.e., conformal) torsion-free connection $D$ such that the symmetric trace-free part of the Ricci tensor of $D$ vanishes. When $D$ is the Levi-Civita connection of a compatible Riemannian metric then this metric is Einstein. As with Einstein metrics, the two dimensional story is somewhat exceptional. A conformal surface with compatible torsion-free connection $D$ is said to be Einstein-Weyl [1] if and only if



where $\operatorname{div}^{D}=\operatorname{tr} D$ is the divergence on 2-forms, $F^{D}$ is the Faraday 2-form of $D$, which is the curvature of $D$ on a natural real line bundle $L^{1}$, and scal ${ }^{D}$ is the scalar curvature of $D$ viewed as a section of $L^{-2}:=\left(L^{1}\right)^{*} \otimes\left(L^{1}\right)^{*}$. If $F^{D}=0$, then $D$ is locally the Levi-Civita connection of a metric of constant scalar curvature.

The idea of studying the two dimensional case was first suggested in [7], in which Pedersen and Tod proposed the goal of classifying the compact examples. This classification was carried out in [1]. Pedersen and Tod also claimed that the local solutions should depend on a single holomorphic function of one variable. The main aim of this paper is to show that this is true for the definition above and to obtain all the solutions explicitly in terms of this holomorphic function.

TheOREm 1.1. Let D be an Einstein-Weyl structure in two dimensions. Then there is a local complex coordinate $\zeta=x+i y$ and a holomorphic function $h$ such that $D=D^{g}+\omega$, where $g=d x^{2}+d y^{2}$ is the flat metric and

$$
\omega=\frac{1}{\bar{h}-\zeta} d \zeta+\frac{1}{h-\bar{\zeta}} d \bar{\zeta} .
$$

The notation used in this paper follows [1]. In particular $L^{w}$ is the real line bundle associated to the representation $A \mapsto|\operatorname{det} A|^{w / 2}$ of $G L_{2}(\mathbb{R})$, so that $L^{-2}$ may 
be identified with $\Lambda^{2} T^{*} M$ once an orientation is chosen. A conformal structure on $M$ may be viewed as a metric on $T M$ with values in $L^{2}$. A Weyl derivative is a covariant derivative $D$ on $L^{1}$. Each choice of compatible metric $g$ trivialises $L^{1}$. If the corresponding trivial Weyl derivative is denoted by $D^{g}$, then $D=D^{g}+\omega$ for some connection 1-form $\omega$. It is well known that Weyl derivatives on a conformal manifold correspond bijectively to compatible torsion-free connections. For instance, $D^{g}$ corresponds to the Levi-Civita connection of $g$.

I prove Theorem 1.1 in Section 2. In the following sections I discuss the extent to which the solutions are genuinely distinct Einstein-Weyl structures, explain the geometry behind the solutions and show how the compact examples arise when $h$ is a (possibly degenerate) Möbius transformation. I end the paper, with a brief discussion of the "twistor theory" of Möbius structures.

2. Local solution of the two dimensional Einstein-Weyl equations. The two dimensional Einstein-Weyl condition is, a priori, nonlinear, but may in fact be linearised. In order to do this I shall make use of the relationship between Weyl structures and Möbius structures [1].

Definition 2.1. A Möbius structure on a conformal manifold $M$ is a (smooth) second order linear differential operator $\mathcal{H}$ from $L^{1}$ to $S_{0}^{2} T^{*} M \otimes L^{1}$ such that for some Weyl derivative $D$, the operator $\mathcal{H}-\operatorname{sym}_{0} D^{2}$ is zero order.

A Möbius structure is a possibly non-integrable and unoriented version of a complex projective structure. More precisely, a Möbius structure $\mathcal{H}$ possesses a tensorial invariant $C^{\mathcal{H}} \in \mathrm{C}^{\infty}\left(M, L^{-2} \otimes T^{*} M\right)$ called the Cotton-York tensor of $\mathcal{H}$, by analogy with the three dimensional case. The Möbius structure is integrable (i.e., given locally by the trace-free Hessian in a suitable chart) if and only if $C^{\mathcal{H}}=0$ (see [1]). In this case, if $M$ is oriented and $\phi$ is a local orientation preserving conformal diffeomorphism then $\phi^{*} \mathcal{H}-\mathcal{H}$ can be identified with the Schwarzian derivative of $\phi$, and so the Möbius structure defines a complex projective structure.

In general the Cotton-York tensor of $\mathcal{H}$ may be computed using an arbitrary Weyl derivative $D$. The result is as follows:

$$
C^{\mathcal{H}}=\operatorname{div}^{D}\left(r_{0}^{D}-\frac{1}{4} s c a l^{D} i d+\frac{1}{2} F^{D}\right),
$$

where $r_{0}^{D}=\mathcal{H}-\operatorname{sym}_{0} D^{2}$. From this, the following result is immediate.

Proposition 2.2. A Weyl structure D in two dimensions is Einstein-Weyl if and only if the trace-free Hessian $\operatorname{sym}_{0} D^{2}$ is locally the trace-free Hessian in some conformal chart.

Consequently, if $D$ is Einstein-Weyl, there is locally a flat metric $g$ such that $\operatorname{sym}_{0} D^{2}=\operatorname{sym}_{0}\left(D^{g}\right)^{2}$. If $D=D^{g}+\omega$, then $\operatorname{sym}_{0} D^{g} \omega-\omega \otimes_{0} \omega=0$. Solving this will give all local solutions of the Einstein-Weyl equation.

Although this equation is still nonlinear, its resemblance to the Riccati equation suggests a way of linearising it. To do this, let $\zeta$ be a local complex coordinate such that $g=d \zeta d \bar{\zeta}$ and write $\omega=f d \zeta+\bar{f} d \bar{\zeta}$ for some complex-valued function $f$. Then the equation for $\omega$ becomes $f^{\prime}=f^{2}$, where $f^{\prime}$ denotes the complex linear part of $d f$. This 
is the Riccati equation if $f$ is holomorphic. Substituting $f=-u^{-1} u^{\prime}$ (which is always possible locally) gives $u^{\prime \prime}=0$ and so $u^{\prime}=\bar{h}_{0}$, for some holomorphic function $h_{0}$. Hence $u=\bar{h}_{0}(\zeta-\bar{h})$, where $h$ is also holomorphic, and so $f=-u^{-1} u^{\prime}=1 /(\bar{h}-\zeta)$.

This proves Theorem 1.1.

3. Gauge transformations. In order to show that the Einstein-Weyl solutions of Theorem 1.1 depend in an essential way on a single holomorphic function, it is necessary to ask to what extent the solutions are equivalent under a change of complex coordinate $\zeta$.

An initial observation is that the scalar curvature and Faraday curvature of $D$ are given by the real and imaginary parts of $h^{\prime} /(h-\bar{\zeta})^{2}$. In particular, $D$ is flat if and only if $h$ is constant, and so most of the solutions are non-trivial.

More generally, note that the complex coordinate $\zeta$ has been partially fixed by requiring that the trace-free Hessian induced by this coordinate chart is the Möbius structure determined by $D$. Hence, the only remaining freedom in $\zeta$ is the freedom to apply Möbius transformations.

If $\zeta=\phi(z)=(a z+b) /(c z+d)$ with $a d-b c \neq 0$, then

$$
d \zeta d \bar{\zeta}=\left|\frac{a d-b c}{(c z+d)^{2}}\right|^{2} d z d \bar{z} .
$$

After rescaling the metric, the Einstein-Weyl structure is given by the new holomorphic function $\tilde{h}=\bar{\phi}^{-1} \circ h \circ \phi$, where $\bar{\phi}(z)=(\bar{a} z+\bar{b}) /(\bar{c} z+\bar{d})$. Thus the EinsteinWeyl structure determines $\bar{h}$ up to conjugation by a Möbius transformation.

4. Geometry of Weyl connections. The transformation law for $\bar{h}$ may be traced back to the fact that it defines a Weyl derivative $D$. If $J^{1} L^{1}$ denotes the bundle of 1-jets of $L^{1}$, then $D$ is a section of the affine subbundle $A(M)$ of $L^{-1} \otimes J^{1} L^{1}$ given by the splittings of the 1-jet projection $J^{1} L^{1} \rightarrow L^{1}$. This affine bundle is modelled on $T^{*} M$.

A Möbius structure on $M$, as a second order linear differential operator on $L^{1}$, defines a vector subbundle $E(M)$ of the 2 -jet bundle $J^{2} L^{1}$. Since this operator is given in coordinates by the trace-free Hessian plus a zero order term, the 1-jet projection $E(M) \rightarrow J^{1} L^{1}$ is surjective, and its kernel, which is the intersection of $E(M)$ with $S^{2} T^{*} M \otimes L^{1}$, is the line bundle $L^{-1}$, embedded as the trace-like tensors.

If $\mu$ is a nonvanishing section of $L^{1}$ and $g$ is the compatible metric corresponding to this trivialisation, then $s c a l^{g} \mu^{2}$ is a function whose value at $x$ depends quadratically on $\left(j^{2} \mu\right)_{x}$. This turns out to define a natural metric of signature $(3,1)$ on $E(M)$ such that the distinguished line $L^{-1}$ is null and is the only null line in the kernel of the projection from $E(M)$ to $L^{1}$ (see [1], and also [3] for more details in the analogous higher dimensional case). Consequently, there is a natural sphere bundle $S^{2}(M)$ over $M$, namely the space of null lines in $E(M)$, and this sphere bundle has a distinguished section. The complement of this section is an affine bundle and this affine bundle is canonically isomorphic to $A(M)$ by projecting each null line into $J^{1} L^{1}$. Therefore a Weyl connection is a section of $S^{2}(M)$ that does not meet the distinguished section.

Now suppose that the Möbius structure is integrable. Then $E(M)$ also possesses a canonical flat connection compatible with the Lorentzian structure. This flat 
connection identifies $S^{2}(M)$ locally with $M \times S^{2}$, and the distinguished section gives the developing map from (open subsets of) $M$ to $S^{2}$. A complex coordinate $\zeta$ on $M$ compatible with the Möbius structure identifies this sphere of parallel sections with $\mathbb{C} \cup\{\infty\}$, so that $\zeta$ itself corresponds to the distinguished section of $S^{2}(M)$. The function $\bar{h}$ arising in Theorem 1.1 is therefore the local coordinate representation of an antiholomorphic section of $S^{2}(M)$. The expression $1 /(\bar{h}-\zeta)$ may be viewed as stereographic projection from $S^{2}(M)$ onto $A(M)$. It is well defined for $\overline{h(\zeta)} \neq \zeta$ and sends poles of $h$ to the origin of $A(M)$ determined by the Levi-Civita connection of $g$.

In fact, if the Weyl connection $D$ is viewed as a section of $A(M)$, its covariant derivative (as a section of $T^{*} M \otimes V(A(M))=T^{*} M \otimes T^{*} M$ ) can be identified with $r_{0}^{D}+\frac{1}{4}$ scal $^{D} i d-\frac{1}{2} F^{D}$, where $r_{0}^{D}=\mathcal{H}-\operatorname{sym}_{0} D^{2}$ (cf. [3]). Hence $D$ is holomorphic if and only if it is flat, and antiholomorphic (with respect to $\mathcal{H}$ ) if and only if $\mathcal{H}=\operatorname{sym}_{0} D^{2}$. The apparent nonlinearity of the Einstein-Weyl condition arises from the fact that the flat connection on $A(M)$ is not affine. Nevertheless, it identifies $A(M)$ locally with an open subset of $M \times S^{2}$, and so the condition for a section to be antiholomorphic is in fact linear.

5. The compact examples. In [1], the local forms of the Einstein-Weyl structures on compact surfaces were found. In this section I shall show that these solutions are obtained when $h$ is a (possibly degenerate) Möbius transformation.

The solutions are given explicitly in terms of a compatible metric and connection 1-form as follows:

where

$$
\begin{aligned}
g & =P(v)^{-1} d v^{2}+v^{2} d t^{2}, \\
\omega & =A v^{2} d t, \\
P(v) & =-A^{2} v^{4}+B v^{2}+C,
\end{aligned}
$$

and $A, B, C$ are arbitrary constants, constrained only by the condition that $P(v)$ should be somewhere positive. In [1], I showed that these Einstein-Weyl structures are defined on $S^{2}$ (for $C>0$ ) or $T^{2}$ (for $C<0$ ) by writing $v$ as an elliptic function of $x$ so that $v^{\prime}(x)^{2}=P(v)$. If instead, one substitutes $v^{2}=1 / u$ and rescales $g$ and $t$ by 2 , then the Einstein-Weyl structure becomes

$$
\begin{aligned}
g & =\frac{1}{u}\left(\frac{d u^{2}}{-A^{2}+B u+C u^{2}}+d t^{2}\right), \\
\omega & =\frac{A d t}{2 u} .
\end{aligned}
$$

Now for $C>0$ introduce a new coordinate $r$ by $u^{\prime}(r)^{2}=\left(-A^{2}+B u+C u^{2}\right) /\left(C r^{2}\right)$. This is readily integrated to give

$$
u(r)=\frac{\left(B^{2}+4 A^{2} C\right)-2 B r^{2}+r^{4}}{4 C r^{2}} .
$$

Rescaling, so that the metric is $d r^{2}+r^{2} d t^{2}$ leads to the solution of Theorem 1.1 given by $h(\zeta)=(B-2 i A \sqrt{C}) / \zeta$. Notice that $\overline{h(\zeta)}=\zeta$ if and only if $\zeta \bar{\zeta}=(B-2 i A \sqrt{C})$. Hence if $A \sqrt{C} \neq 0$, the solution is globally defined on $S^{2}$. 
For $C<0$ introduce instead a coordinate $\theta$ by $u^{\prime}(\theta)^{2}=\left(-A^{2}+B u+C u^{2}\right) /(-C)$. This integrates to give

$$
u(\theta)=\frac{B+\sqrt{B^{2}+4 A^{2} C} \sin \theta}{-2 C}
$$

and the Einstein-Weyl structure becomes

$$
\begin{aligned}
& g=\frac{1}{B+\sqrt{B^{2}+4 A^{2} C} \sin \theta}\left(d t^{2}+d \theta^{2}\right), \\
& \omega=\frac{A \sqrt{-C} d t}{B+\sqrt{B^{2}+4 A^{2} C} \sin \theta},
\end{aligned}
$$

which is globally defined on $T^{2}$ (for $C<0$ and $B^{2}+4 A^{2} C>0$ ). After rescaling so that the metric is $e^{2 t}\left(d t^{2}+d \theta^{2}\right)$, the solution $h(\zeta)=i(B+2 A \sqrt{-C}) \zeta / \sqrt{B^{2}+4 A^{2} C}$ of Theorem 1.1 is obtained.

More generally if $\bar{h}$ is an orientation reversing Möbius transformation, then the Weyl connection is well defined away from the fixed points of this transformation. Hence the elliptic elements, apart from the simple inversions (which have an invariant circle), give solutions globally defined on $S^{2}$ (equivalent to one of the solutions above). The hyperbolic elements, with two fixed points, correspond to the solutions on $T^{2}$ (they are periodic solutions on a cylinder). The remaining cases occur as limits. For instance the simple inversions, such as $\zeta \mapsto 1 / \bar{\zeta}$, give the hyperbolic metric.

6. Twistor theory. The twistor space of a conformal 2-manifold $M$ is its orientation double cover, viewed as a complex curve $\Sigma$. This is a rather trivial two dimensional analogue of the four dimensional theory (see, for instance, [4]). Note that $\Sigma$ has a real structure given by the nontrivial involution in each fibre and that $M$ may be recovered from $\Sigma$ as the moduli space of real pairs of points. The full moduli space of (unordered, distinct) pairs of points in $\Sigma$ is $M^{\mathbb{C}}=(\Sigma \times \Sigma \backslash \Delta(\Sigma)) / S_{2}$. This complex surface has a natural conformal structure: a tangent vector to $M^{\mathbb{C}}$ at $\left\{x_{1}, x_{2}\right\}$ consists of a pair of tangent vectors to $\Sigma$ (at $x_{1}$ and $x_{2}$ ), and it is null if one of these components vanishes. Hence $\Sigma$ is (locally) the space of null geodesics in $M^{\mathbb{C}}$. Of course $M^{\mathbb{C}}$ is the natural space in which real analytic functions on $M$ may be written $f=f(z, \bar{z})$ with $f$ holomorphic in two variables.

Although this notion of twistor space has no real content, it does provide a formal way to distinguish an integrable Möbius structure in two dimensions from a one dimensional complex projective structure. The former is a trace-free Hessian $L^{1} \rightarrow S_{0}^{2} T^{*} M \otimes L^{1}$, whereas the latter is a second order operator $\mathcal{L} \rightarrow\left(T^{*} \Sigma\right)^{2} \otimes \mathcal{L}$ (on a line bundle $\mathcal{L}$ with $\mathcal{L}^{2}=T \Sigma$ ) whose symbol is the identity. The two are easily related: $\left(T^{*} \Sigma\right)^{2}$ is the pullback of $S_{0}^{2} T^{*} M$, and since $T \Sigma \otimes \overline{T \Sigma}$ is (the pullback of) $L^{2} \otimes \mathbb{C}$, it follows that $\mathcal{L} \otimes \overline{\mathcal{L}}$ can be identified with $L^{1} \otimes \mathbb{C}$. The projectivisation of $J^{1} \mathcal{L}$ corresponds to $S^{2}(M)$, and the complex projective structure defines a connection $J^{1} \mathcal{L} \rightarrow J^{2} \mathcal{L} \leq J^{1}\left(J^{1} \mathcal{L}\right)$ that projectivises to the flat connection on $S^{2}(M)$ induced by the integrable Möbius structure.

A more satisfying twistorial description would encode the Möbius structure in pure holomorphic geometry. Nevertheless, I hope the naïve twistor theory given here at least provides some light entertainment. 


\section{REFERENCES}

1. D. M. J. Calderbank, Möbius structures and two dimensional Einstein-Weyl geometry, J. Reine Angew. Math. 504 (1998), 37-53.

2. D. M. J. Calderbank and H. Pedersen, Einstein-Weyl geometry, Edinburgh Preprint MS-98-010 (1998), to appear in Essays on Einstein manifolds (International Press, 1999), 387-403.

3. P. Gauduchon, Connexion canonique et structures de Weyl en géométrie conforme, Preprint (1990).

4. P. Gauduchon, Structures de Weyl-Einstein, espaces de twisteurs et variétés de type $S^{1} \times S^{3}, J$. Reine Angew. Math. 469 (1995), 1-50.

5. N. J. Hitchin, Complex manifolds and Einstein equations, Twistor geometry and non-linear systems (eds H. D. Doebner and T. D. Palev), Lecture Notes in Math. No 970 (Springer-Verlag, 1982), 79-99.

6. P. E. Jones and K. P. Tod, Minitwistor spaces and Einstein-Weyl spaces, Classical Quantum Gravity 2 (1985), 565-577.

7. H. Pedersen and K. P. Tod, Three-dimensional Einstein-Weyl geometry, $A d v$. Math. 97 (1993), 74-109. 